\title{
Intensity effects of the auditory evoked brain response to stimulus onset and cessation
}

\author{
PAULA KLINGENBERG SCHWEITZER* and DONALD I. TEPAS \\ Saint Louis University, St. Louis, Missouri 69103
}

\begin{abstract}
Average evoked brain responses (EBR) to the onset (ON) and cessation (OFF) of $1-\mathrm{kHz}$ pure-tone stimuli were computed from human scalp recordings. Stimuli of $2,000 \mathrm{msec}$ duration were presented binaurally at 10 intensity levels. The waveform of the OFF EBR is similar to the ON EBR. The ON response is in general larger than the OFF response. Comparable ON and OFF amplitude response measures are both sensitive to changes in stimulus intensity which may be fit by a linear function. Significant differences in slope between $\mathrm{ON}$ and $\mathrm{OFF}$ amplitude intensity functions were demonstrated, which suggests different physiological systems for these responses.
\end{abstract}

The human averaged evoked brain response (EBR) has been shown to be a sensitive measure reflecting changes in acoustic stimulation. Most of the research on the auditory EBR has been concerned with changes following stimulus onset (ON response), while relatively little has been devoted to changes following stimulus cessation (OFF response). The ON response shows linear amplitude-intensity functions when appropriate conditions and measurements are used (Tepas, Boxerman \& Anch, 1972). With regard to the OFF response, the relationship of EBR measures and intensity is not clear.

Early studies on the OFF EBR (Keidel, 1963-64; Rose \& Malone, 1965) fail to clearly specify either stimulus parameters or response measures. In addition, no comparison of ON and OFF EBR measures was reported in either study. More recent studies compare the ON and OFF measures and show some differences. The latency of the OFF response has been reported to be similar to that of the ON response (Spychala, Rose, \& Grier, 1969; Onishi \& Davis, 1968), but there is some evidence that at least one component of the OFF response may have a shorter latency (Onishi \& Davis, 1968; Johannsen, Keidel, \& Spreng, 1972). Amplitude comparisons have shown the OFF response to be smaller than the ON response (Spychala et al, 1969; Onishi \& Davis, 1968; Johannsen et al, 1972).

A general trend toward increasing amplitude and decreasing latency of the OFF response has been suggested by some authors (Onishi \& Davis, 1968; Johannsen et al, 1972). Only three or four stimulus intensities were used in each of these studies. These minimal manipulations of stimulus inten-

*Requests for reprints should be sent to Paula Klingenberg Schweitzer, Department of Psychology, St. Louis University, 221 N. Grand Blvd., St. Louis, Missouri 63103. sity do not allow accurate assessment of stimulus magnitude effects. It should also be noted that only a small number of potentials was averaged in these studies. This may have diminished the reliability of measuring the small responses produced by low-magnitude stimulation. Thus, the specific relationship of amplitude and latency to stimulus intensity remains unclear for the OFF response. In addition, the relationship of the OFF EBR to the ON EBR remains inconclusive. The purpose of the present study is to clarify these relationships, by systematically investigating the effects of intensity on the human EBR to the onset and to the offset of auditory stimulation.

\section{METHOD}

\section{Stimulus Generation Apparatus}

The general methodology and recording technique was similar to that reported by Tepas (1974) and Tepas et al (1972). One-kilohertz pure tones were presented binaurally to the $S$ at a rate of 1 every 4 sec. A Tektronix pulse generator was used to trigger a series of Grason-Stadler timers, as well as to control AX08 peripheral input to the LAB-8/1 computer system of the Digital Equipment Corporation at a rate of 1 every $2 \mathrm{sec}$. The duration of the stimulus was $2,000 \mathrm{msec}$. The timers were set up to control a pair of Clare $\mathrm{Hg} 1004$ mercury wetted relays. These relays functioned in conjunction with the sync pulse output from the pulse generator in controlling averaging of the analog input to the AX08 laboratory peripheral. By changing the AX08 contingency register, closure of one relay permitted separate storage of the data for the ON response while closure of the second relay permitted separate storage of the data for the OFF response. The timers provided automatic control of the AX08 contingency register changes. Grason-Stadler attenuators regulated the intensities of the stimuli. Ten different intensities, ranging from 54 to $90 \mathrm{~dB}$ SPL (re .0002 dynes $/ \mathrm{cm}^{2}$ ) were used. Thus, stimulus intensity was varied in 4-dB steps. An electronic switch which controlled the $10-\mathrm{msec}$ rise-fall time was interfaced with these modules.

The stimuli were delivered through matched Telephonics TDH 39 earphones mounted in MX41/AR cushions and an HD11B headset. The earphones were calibrated with a Bruel and Kjaer Model 158 audiometer calibrator with an NBS 9A 6cc acoustic coupler. 


\section{Recording Apparatus}

The electroencephalograph (EEG) was recorded on one channel of a Grass Model 7 polygraph. The Model 7P5A EEG preamplifier was set at a one-half amplitude high-frequency band pass of $500 \mathrm{~Hz}$, while the time constant of the low-frequency band pass was .45 . The 60-cycle filter on the Model $7 \mathrm{P}$ ac driver amplifier was not used. The EEG was recorded by means of Grass E5S electrodes placed on the scalp at $\mathrm{C}_{Z}$ and $\mathrm{O}_{Z}$ (Jasper, 1958). A Beckman 16-mm biopotential electrode attached to the forehead served as ground.

The AX08 analog input of the LAB-8 computer system received the amplified EEG, and averaging was accomplished using the Advanced Averager Program (DEC-DB-U18C-PB). EBR computations were made at 500 time points distributed over a $500-\mathrm{msec}$ analysis time starting at either stimulus onset or offset. Each EBR was the sum of the EEG activity following 70 of these stimulus presentations or removals. One trial consisted of 70 of the stimulus presentations or removals. Both ON and OFF EBR waveforms were computed concurrently on each trial. The EBRs computed were displayed on an oscilloscope, and the digital values for each of the 500 time points were punched on paper tape for subsequent analysis off-line.

\section{Subjects}

Three adult male volunteers, ranging in age from 22 to 26 years, served as Ss. Ss were selected on the basis of their lack of any obvious hearing deficits and their ability to clearly produce a measurable OFF EBR, since there is no definitive information in the literature as to the appropriate conditions for detecting a reliable OFF response.

\section{Procedure}

Each $S$ was tested on 12 sessions. ON and OFF EBRs to each of the 10 intensities were obtained during each session with only 1 intensity being administered per trial. The order of presentation of the stimuli was randomized across the 10 trials for each of the 12 sessions for each $\mathbf{S}$. Ss were not informed of the order used. After all 12 sessions were completed, the punched paper tapes containing the digital values of the EBR for separate trials were summed together across sessions for each $S$ at each intensity level. This resulted in one waveform for the 840 stimulus presentations or removals for each stimulus intensity for each $S$. The summed waveforms were then printed out on an ASR 33 Teletype as decimal values. Amplitude and latency measures were made from these decimal values. A standard calibration square wave $(10 \mu \mathrm{V}$ and $100 \mathrm{msec})$ was run though the system and averaged at the beginning and end of each session, then summed across sessions to provide a scale for measurement of these values.

\section{RESULTS}

Figure 1 illustrates the typical ON and OFF EBR for S K.F. Peak deflections were labeled in accordance with the nomenclature system used in previous studies from this laboratory (Tepas. 1974). These deflections are identified in Fig. 2 as A, B, C, $D$, and $E$ for the ON EBR, and F, G, H, and $I$ for the OFF EBR. For each ON EBR, the following measures were made: Amplitudes B-C, C-D, and D-E; Peak Latencies B, C, D, and E. For each OFF EBR, the following measures were made: Amplitudes F-G, G-H, and H-I; Peak Latencies F, G, H, and I.

Figure 2 shows the summed ON and OFF EBRs for S K.F. at each intensity. The data shown are representative for all Ss. As the figure indicates, the amplitude of the OFF EBR was consistently smaller than that of the ON EBR. In addition, it can be seen that there is some similarity in waveform of the two responses. Some components of the OFF response $(H$ and I) appear to have a latency range similar to that of
SUBJECT: $K F$

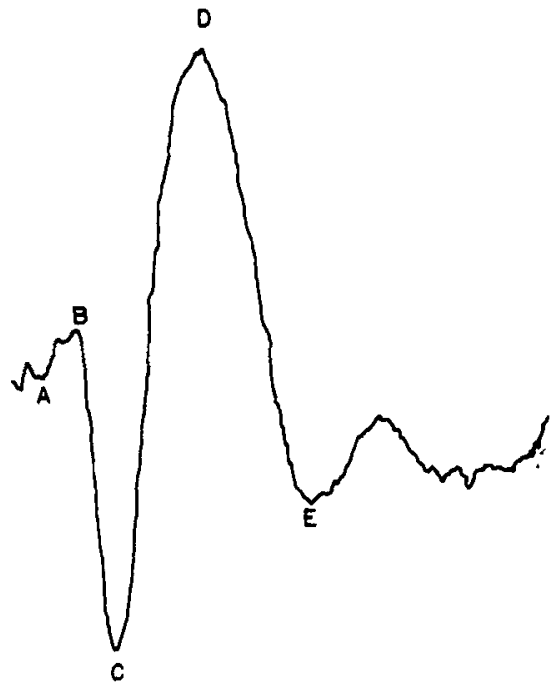

90 dB SPL

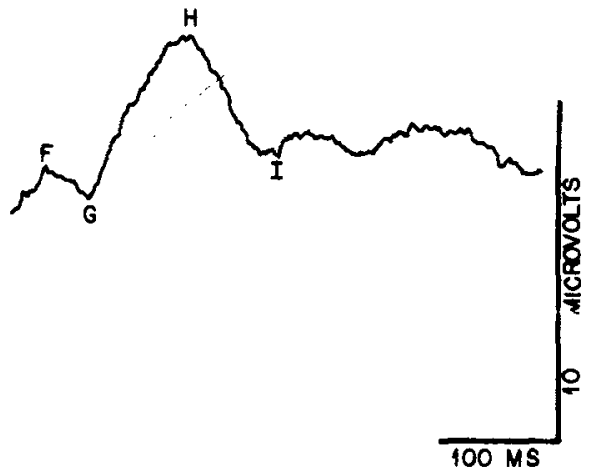

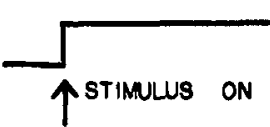

pstimulus off

Fig. 1. EBR to the onset and offset of acoustic stimulation for S K.F. Stimulus duration is 2 sec. Downward deflection indicates that the $O_{z}$ electrode was positive with respect to the $C_{z}$ electrode. Each waveform is the sum of 12 trials. 


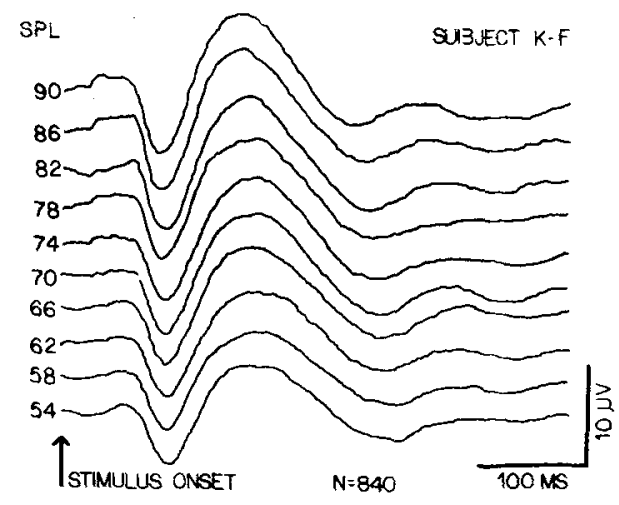

Table 1

Correlations of EBR Amplitude and Latency Measures With SPL for the ON and OFF Responses for Subjects KF, RD, and JS and Data Pooled Across Subjects

\begin{tabular}{ccccc}
\hline Measure & $\mathrm{KF}$ & $\mathrm{RD}$ & $\mathrm{JS}$ & Pooled \\
\hline $\mathrm{B}-\mathrm{C}$ & $.945^{* *}$ & .213 & .104 & - \\
$\mathrm{C}-\mathrm{D}$ & $.969^{* *}$ & $.941^{* *}$ & $.838^{* *}$ & $.971^{* *}$ \\
$\mathrm{D}-\mathrm{E}$ & $.848^{* *}$ & $.951^{* *}$ & $.690^{*}$ & $.986^{* *}$ \\
F-G & - & - & - & - \\
G-H & $.918^{* *}$ & $.897^{* *}$ & $.958^{* *}$ & $.986^{* *}$ \\
H-I & $.719^{*}$ & -.175 & -.365 & - \\
B & .107 & -.557 & -.541 & - \\
C & $-.865^{* *}$ & $-.889^{* *}$ & $-.932^{* *}$ & $-.825^{* *}$ \\
D & -.613 & $-.683^{*}$ & .168 & - \\
E & $-.961^{* *}$ & .284 & $-.881^{* *}$ & - \\
F & - & - & - & - \\
G & $-.745^{*}$ & $-.911^{* *}$ & -.405 & $-.930^{* *}$ \\
H & $-.768^{*}$ & -.323 & $-.812^{*}$ & - \\
I & -.597 & .058 & -.112 & - \\
\hline$* 632=p$ & $<.05$ & $* * .765=p<.01$ &
\end{tabular}

the $\mathrm{ON}$ response, while other components (F and $\mathrm{G}$ ) do not. In addition, Component $F$ could not be reliably measured under all conditions.

As a test for linearity of amplitude-intensity and latency-intensity relationships, linear regression lines were computed by the method of least squares, and correlation coefficients were calculated. These correlation coefficients and their significance levels are shown in Table 1. The regression analysis revealed highly significant correlations between amplitude and SPL for each S for Measure C-D of the ON EBR and Measure G-H of the OFF EBR. The regression lines and mean data points for these measures are presented in Fig. 3 for each S, along with the slopes of the best fit lines. Linear regression analysis on the data pooled across Ss also showed significant

Fig. 2. ON AND OFF EBRs for S K.F. Note the different microvolt calibration for the two figures. Each waveform is the sum of 12 trials.

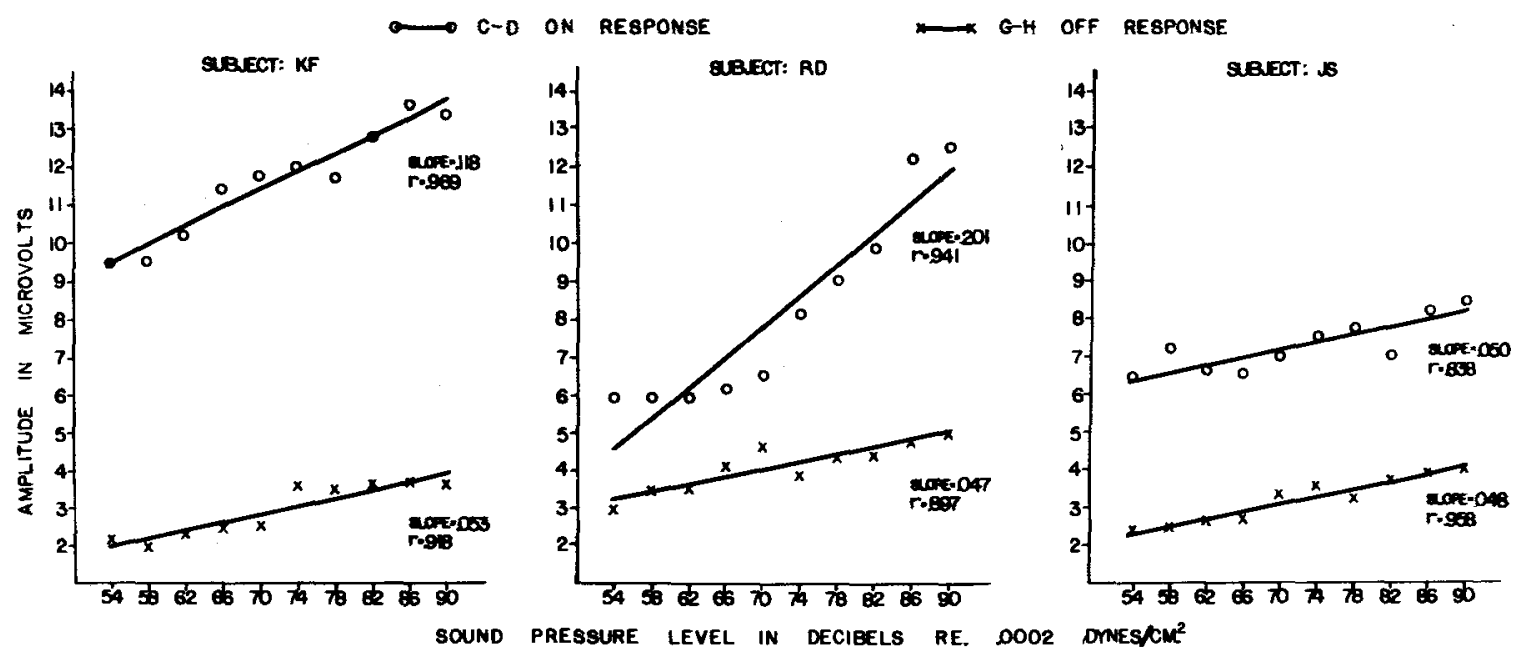

Fig. 3. EBR amplitude-intensity functions for the ON (C-D) and OFF (G-H) responses for each S. The regression lines were fit to the mean data points using the method of least squares. Measurements were made from the digital values of the waveforms. 
POOLED DATA

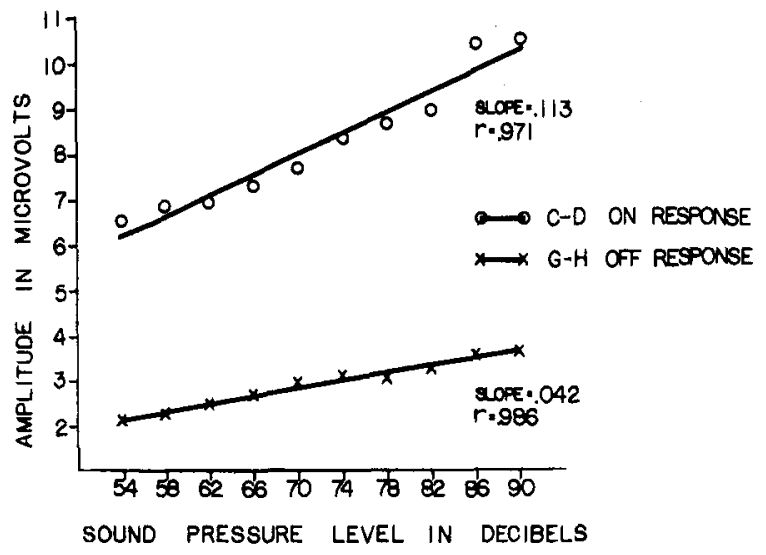

Fig. 4. EBR amplitude-intensity functions for the $O N$ (C-D) and OFF (G-H) responses for data pooled across Ss. Measurements were made from the digital values of the waveforms. correlation coefficients for Measures C-D and G-H. Figure 4 shows the regression lines and mean data points for these pooled data. The slopes for these functions were $.113 \mu \mathrm{V} / \mathrm{dB}$ for $\mathrm{C}-\mathrm{D}$ and $.042 \mu \mathrm{V} / \mathrm{dB}$ for $\mathrm{G}-\mathrm{H}$.

Significant negative correlations between latency and SPL for each $\mathrm{S}$ as well as the pooled data are seen for Measure $C$. The OFF response data for the corresponding measure, $G$, are not as conclusive, as only two of the three Ss showed significant correlation coefficients. The latency-intensity regression lines for $\mathrm{C}$ and $\mathrm{G}$ for each $\mathrm{S}$ are shown in Fig. 5, along with the slopes of the regression lines.

A significant difference between the slopes (McNemar, 1969) of the ON and OFF amplitudeintensity functions was revealed for Ss K.F. and R.D. and for the pooled data. For S J.S. there was no significant difference between the two slopes. A significant difference between the slopes of the ON

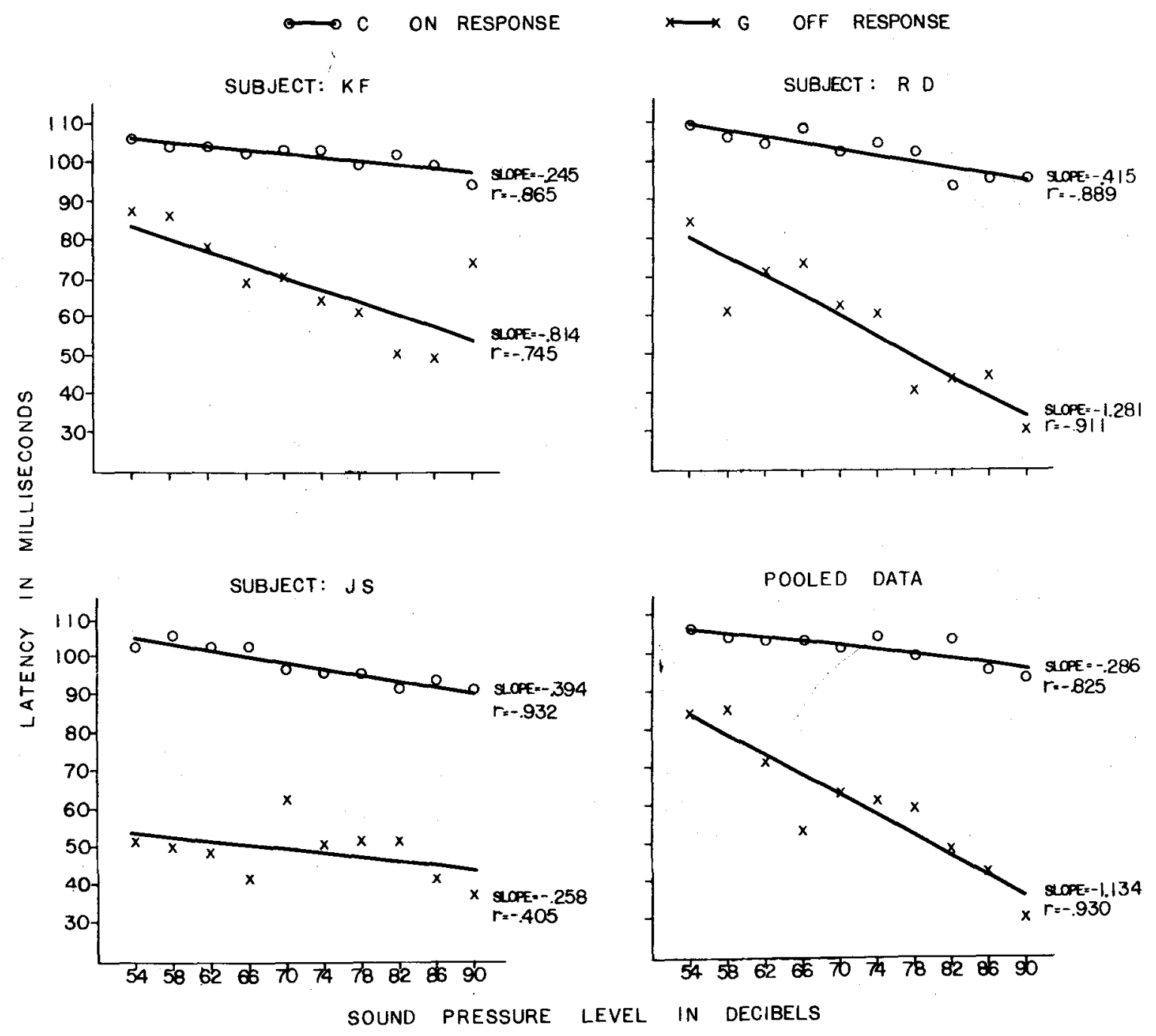

Fig. 5. EBR latency-intensity functions for the $\mathrm{O}^{\prime}(C)$ and OFF (G) responses for each $S$ and for data pooled across Ss. Measurements were made from the digital values of the waveforms. 
and OFF latency-intensity functions was also revealed for all data except that of S J.S.

\section{DISCUSSION}

The results of this study show that an OFF EBR reliably occurs following the cessation of a $2,000-\mathrm{msec}$ stimulation for the Ss under the conditions used in the present study. The OFF response is comparable to the ON response, with similar major deflections present in both responses. Components $\mathrm{C}, \mathrm{D}$, and $\mathrm{E}$ of the $\mathrm{ON}$ EBR have clearly evident counterparts in $\mathrm{G}, \mathrm{H}$, and I of the OFF EBR. The finding that $F$ was difficult to detect supports the data of Onishi and Davis (1968), who indicated a difficulty in detecting components comparable to $F$ and $G$. The OFF EBR is of smaller amplitude than the ON EBR, supporting the data of Onishi and Davis (1968), Spychala et al (1969) and Johannsen et al (1972).

Most importantly, both the ON and the OFF responses are sensitive to changes in the intensity of stimulation. This sensitivity, however, is dependent on the selection of the appropriate EBR measure and the findings are consistent with $\mathrm{ON}$ data reported by Tepas et al (1972). Linear amplitude-intensity functions were demonstrated for corresponding $O N$ and OFF Response Measures C-D and G-H. The increase in amplitude with intensity was not as steep for the OFF response as for the ON response. Thus, appropriate $\mathrm{ON}$ measures appear to be more sensitive to stimulus intensity change than do similar OFF measures. This slope difference also suggests that the ON and OFF responses may be generated from different, though not necessarily exclusive, physiological mechanisms. If the two responses were generated by the same physiological system, one would expect similar ON and OFF EBR slopes within an individual $S$.

The shorter response latency for G $(30-90 \mathrm{msec})$ as compared to $C(95-110 \mathrm{msec})$ supports the data of Onishi and Davis (1968) and Johannsen et al (1972) and further suggests that the ON and OFF responses are functions of separate physiological mechanisms with different response latencies. Under the conditions of this study, latency of the OFF response is not as sensitive a measure of intensity change as amplitude is. No single OFF response latency measure showed significant correlations with SPL for all three Ss. Latency of the ON response, however, is sensitive to changes in intensity when $C$ is used as the response measure, showing a linear decrease with an increase in SPL.

Given the latency relationships found in this study, it seems possible that ON and OFF responses interact for short-duration stimuli. That is, some components of the OFF response have latencies similar to those of the $O N$ response. If these latencies remain fairly constant with changes in stimulus duration, then ON response data for stimuli less than some critical duration may be confounded by OFF response interaction. Davis and Zerlin (1966) have suggested that a possible interaction between $O N$ and $O F F$ responses could account for the slight amplitude increase they found for the ON response for durations of less than 30 msec. Johannsen et al (1972) have also mentioned the possibility of ON and OFF interactions for short-duration stimuli. A study of ON and OFF EBR interactions, systematically varying stimulus duration and intensity, may help to resolve the question of to what extent the ON and OFF responses share the same physiological mechanism.

\section{REFERENCES}

Davis, H., \& ZerLIN, S. Acoustic relations of the human vertex potential. Journal of the Acoustical Society of America, 1966, 39, 109-116.

JASPER, H. The ten-twenty electrode system of the international federation. Electroencephalography \& Clinical Neurophysiology, $1958,10,371-375$.

Johannsen, H. S., Keidel, W. D., \& Spreng, M. Der Einfluss von Intensitat und Dauer der Beschallung auf den OFF-Effekt des akustisch evozierten Potentials. Archiv fuer klinische und experimentelle Ohren-, Nasen- und Kehlkopfheilkunde, 1972, 201, 208-221.

KEIDEL, W. D. Tuning between central auditory evoked responses using pure tone stimuli. Bionics Symposium, 1963-64, 330, 60.

McNemar, Q. Psychological statistics. New York: Wiley, 1969.

OnISHI, S., \& DAVIs, H. Effects of duration and rise time of tone bursts on evoked $\mathrm{V}$ potentials. Journal of the Acoustical Society of America, 1968, 44, 582-591.

Rose, D., \& Malone, J. Some aspects of the acoustically evoked response to the cessation of stimulus. Journal of Auditory Research, 1965, 5, 27-40.

Spychala, P., Rose, D., \& Grier, J. Comparison of the "ON" and "OFF" characteristics of the acoustically evoked response. International Audiology, 1969, 8, 416-423.

TEPAS, D. I. Computer analysis of the electroencephalogram: Evoking, promoting and provoking. Behavior Research Methods \& Instrumentation, 1974, 6, 95-110.

TePas, D. I., Boxerman, L., \& ANCH, A. M. Auditory evoked brain responses: Intensity functions from bipolar human scalp recordings. Perception \& Psychophysics, 1972, 11, 217-221.

(Received for publication April 19, 1974; revision received June 10,1974 .) 